Article

\title{
Dynamic Tuning of Transmission Wavelength of MEMS-Based Ge Waveguides on a Si Beam
}

\author{
Masashi Hirase, Luan M. Nguyen, Hiroshi Fukuda, Yasuhiko Ishikawa and Kazumi Wada * \\ Department of Materials Engineering, The University of Tokyo, Tokyo 113-8654, Japan; \\ hirase.microphotonics@gmail.com (M.H.); luan.microphotonics@gmail.com (L.M.N.); \\ fukuda.microphotonics@gmail.com (H.F.); y-ishikawa@material.t.u-tokyo.ac.jp (Y.I.) \\ * Correspondence: kwada@material.t.u-tokyo.ac.jp; Tel.: +81-35-841-7141
}

Received: 1 March 2016; Accepted: 17 March 2016; Published: 30 March 2016

\begin{abstract}
Three-dimensional structures of microelectro-mechanical systems (MEMS)-based Ge waveguide on a Si beam were fabricated for dynamic tuning of the fundamental absorption edge of Ge by external stressing. The application of various amounts of external forces up to $1 \mathrm{GPa}$ onto the Si beam shows clear red-shifts in the absorption edge of Ge waveguides on the Si beam by $\sim 40 \mathrm{~nm}$. This shift was reproduced by the deformation potential theory, considering that mode of propagation in the Ge waveguide. The wavelength tuning range obtained makes it possible to cover the whole C-band of optical communication, indicating it to be a promising approach to electro-absorption Ge modulators to get them to work with a broader wavelength range than previously reported.
\end{abstract}

Keywords: modulators; electro-refractive; dynamic tuning, integrated photonics; MEMS

\section{Introduction}

Si photonics is an enabler of electronic and photonic integration on a Si CMOS (complementary metal oxide semiconductor) platform for cost-effective, bandwidth-abundant computation and communication on-chip networks. The current device challenge in Si photonics is the optical modulator to reduce the power consumption. Electro-refraction modulators (ERMs) such as Mach-Zehnder Interferometer (MZI) modulators [1,2] reported high power consumption in the order of $\sim \mathrm{pJ} / \mathrm{bit}$ because of the plasma effect that takes place to achieve the refractive index change of the long waveguide arm. Another type of ERM is ring resonator modulators [3,4], which do not require high power to operate but do require thermal management to match the resonance with the modulation wavelength. In contrast, electro-absorption modulators (EAMs) are essentially free from large power consumption because of the operation under reverse bias. The EAMs reported so far are based on the Franz-Keldysh effect of GeSi and Ge [5-11] and the quantum confined Stark effect of SiGe/Ge quantum wells $[12,13]$. The current challenge in EAMs is to expand their operation wavelength range, i.e., currently only $\sim 10 \mathrm{~nm}$ with a reasonable extinction ratio $(\sim 10 \mathrm{~dB})$. Since the range is limited by the fundamental absorption edge, Ge alloying with $\mathrm{Si}$ [7] and stress-compressive Ge [11] can tune the operation wavelength of EAMs to $1550 \mathrm{~nm}$. However, the range of the operation wavelength remained unchanged in these approaches.

The present paper has demonstrated dynamic tuning of the fundamental absorption edge of Ge waveguides in terms of applying stress in Ge waveguides on a Si beam (cantilever). The stress tuning concept was applied in the three-dimensional microelectro-mechanical systems (MEMS)-based beam structures and the bandgap shifts from applying stress were detected by the photoluminescence peak shifts [14-18]. To apply the concept to expand the range of the operation wavelength in terms of external stress, Ge waveguides are designed to be implemented on a Si waveguide on the Si beam. The devices have demonstrated $\sim 40 \mathrm{~nm}$ red-shifts in the fundamental absorption edge of the Ge waveguides by dynamic external forces up to $1 \mathrm{GPa}$ on the Si beam. The shifts were consistent with 
the deformation potential calculations taking the propagation mode in the Ge waveguide into account. This prototype should be an enabler of the Ge EAMs with reconfigurable wavelength in the whole C-band in terms of external dynamic stressing.

\section{Design of Ge Waveguide to Tune the Fundamental Absorption Edge}

To design the Ge waveguide on a MEMS-based Si beam, the structure should be optimized to meet both optical and mechanical requirements. In this chapter, the design is optimized in terms of controlling the fundamental absorption edge via stress, the structure of the Ge waveguide on the $\mathrm{Si}$ beam, and the mode of propagation.

\subsection{Design of the Fundamental Absorption Edge and Stress}

The dependence of the fundamental absorption edge of the Ge waveguide on one-dimensional stress along the $<110>$ direction is calculated on the basis of deformation potential theory [18] as shown in Figure 1. The reason to choose the direction is related to Ge epigrowth and is discussed later. Built-in two-dimensional tensile stress is accumulated in the Ge epilayer grown on $\mathrm{Si}$, and it splits the valence band into heavy hole $(\mathrm{HH})$ and light hole $(\mathrm{LH})$ even at no $<110>$ stress [14]. Here, $\Gamma-\mathrm{HH}$ $(\Gamma-\mathrm{LH})$ denotes the electron transition from the top of the $\mathrm{HH}(\mathrm{LH})$ band to the bottom of the $\Gamma$ valley. It is clear that both $\Gamma-\mathrm{HH}$ and $\Gamma-\mathrm{LH}$ show "red-shifts" when one-dimensional tensile stress is applied to Ge. This indicates that the fundamental absorption edge of a Ge waveguide should be dynamically tuned depending on the amount of stress applied.

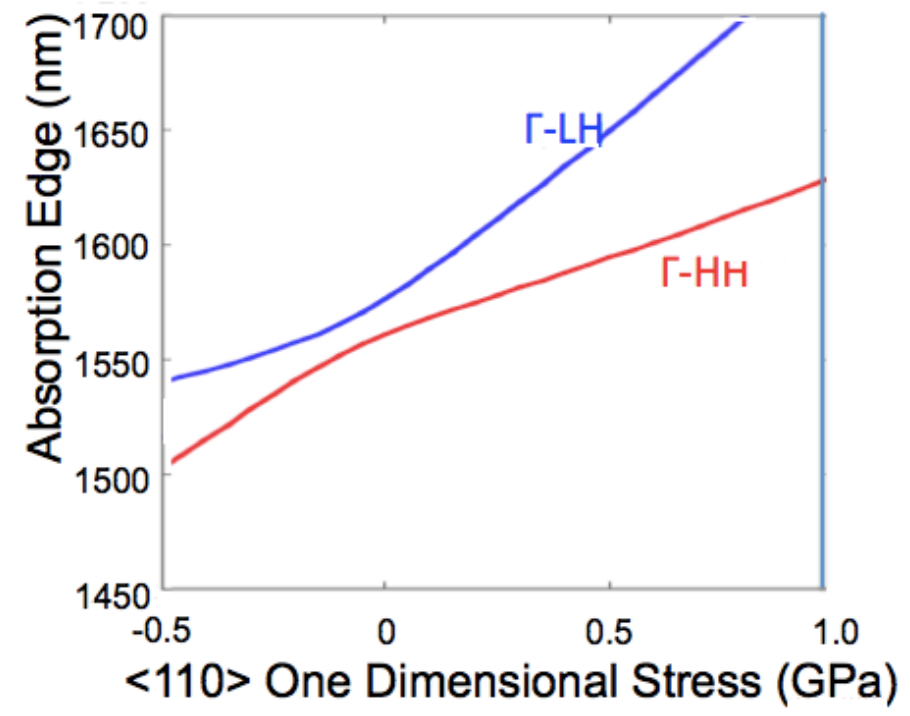

Figure 1. Fundamental absorption edge vs. $<110>$ one-dimensional tensile stress of Ge. Ge has a built-in strain of $0.17 \%$ biaxial tensile due to growth cooling. This generates splitting of heavy hole $(\mathrm{HH})$ and light hole $(\mathrm{LH})$ even when no stress is applied. Applying one dimensional $<110>$ tensile stress to the beam produces red-shifts in the absorption edge. The detailed calculations of the dispersion relation were reported elsewhere [11].

\subsection{Design of Ge Waveguide on Si Beam Structure and Stress}

The structure of the Ge waveguide on the Si beam and its effective stressing are described. Finite domain time difference (FDTD) simulations are used to design single mode Ge waveguides on the beam, as schematically shown in Figure 2: a 5- $\mu \mathrm{m}$-long, 400-nm-thick and 400-nm-wide Ge waveguide on a Si rib waveguide with a $150 \mathrm{~nm}$ rib top on the Si on the insulator (SOI) wafer. The SOI wafer has a 250-nm-thick top Si layer on a 3- $\mu$ m-thick buried oxide (BOX) layer. Here, the Si rib waveguide is employed to mechanically support the waveguide after removing the BOX layer under the Si beam. 
Employing the rib structure makes the bending radius of the Si waveguide large to minimize radiation losses. The 150-nm-high Si rib waveguide allows a bending radius of $10 \mu \mathrm{m}$ which remains lower than $0.1 \mathrm{~dB}$ at the right angle bend, based on mode solver simulations. Based on these optimizations, our design of the Si beam to implement the Si rib waveguide should be $30 \mu \mathrm{m}$ wide and $20 \mu \mathrm{m}$ long. Such a large beam structure requires a specific design for effective stressing of the Ge waveguide. The neck structures as shown in Figure 2a are to enhance the stress to the Ge waveguide on the wide beam. The finite element method simulation of stress is performed to optimize the neck to be $5 \mu \mathrm{m}$ wide and $5 \mu \mathrm{m}$ long in shape and it indicates the amount of tensile stress to the Ge waveguide should be as high as $1 \mathrm{GPa}$ when bent down to the base Si.

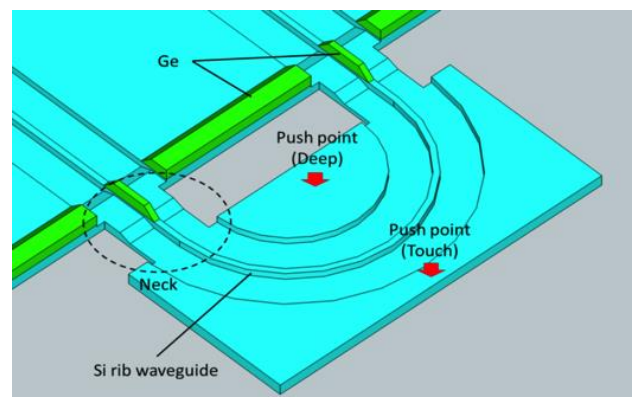

(a)

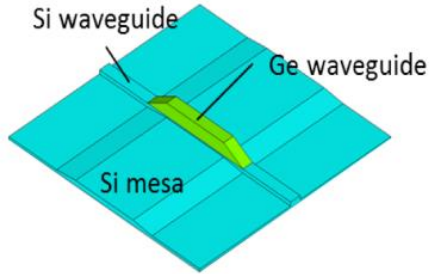

(b)

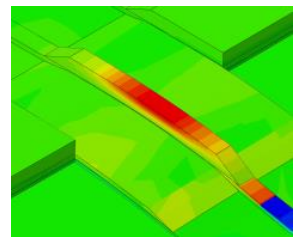

(c)

Figure 2. Schematic diagram of Ge waveguide on Si rib waveguide on Si beam. (a) The beam structure with two necks; (b) Magnified view near the neck region indicated by a dotted circle in (a), and (c) stress distribution by FEM.

The finite element method (FEM) reveals the stress distribution in the Ge waveguide on the neck, as shown in Figure 2c. The stress should have a linear depth profile, and thus the top of the Ge waveguide has the highest stress (red) and the bottom has the lowest (green).

The stresses at the typical point of the Ge waveguide are qualitatively shown in Table 1 by taking reference points such as "Ge top", a half-point of the thickness as "Ge middle", and the interface between Ge and Si as "Ge bottom". Here, an external force is applied at "Touch" and "Deep" until the beam touches down to the base Si. It has a peak near the waveguide direction. Here, the propagation mode would be the TE mode and the mode field is the highest near the Ge middle in steady state. Thus, the calculated amounts of stress at the Ge middle should be 0.2 and $1.3 \mathrm{GPa}$ when applying stress to "Touch" and "Deep" and, needless to say, 0 GPa at "No stress". These stress amounts are taken at the peak point along the wavelength direction. The predicted fundamental absorption edges are calculated, using Figure 1, some of which are shown in Table 1: referencing the Ge middle point at "No stress", the red-shifts of $\Gamma$-HH should be $15 \mathrm{~nm}$ at "Touch" and $90 \mathrm{~nm}$ at "Deep", while those of $\Gamma$-LH should be $35 \mathrm{~nm}$ at "Touch" and $200 \mathrm{~nm}$ at "Deep".

Table 1. Stress accumulated in the Ge waveguide.

\begin{tabular}{cccc}
\hline \multirow{2}{*}{ Stress Application Point } & \multicolumn{2}{c}{$\begin{array}{r}\text { Tensile Stress Amount (GPa) (Absorption Edges (nm) of } \Gamma \text {-HH } \\
\text { and } \Gamma \text {-LH, Calculated from Figure 1) }\end{array}$} \\
\cline { 2 - 4 } & Ge Top & Ge Middle & Ge Bottom \\
\hline No stress & $0(1560,1570)$ & $0(1560,1570)$ & $0(1560,1570)$ \\
\hline Touch & $0.3(1580,1620)$ & $0.2(1575,1605)$ & $0.04(1565,1582)$ \\
\hline Deep & $2.1^{*}$ & $1.31 .3(1650,1770)$ & $0.17(1575,1605)$ \\
\hline
\end{tabular}

*: The ammount of stress 2.1 GPa may be too large to extrapolate it to the bandgap energies. Thus, there are no indications of corresponding wavelengths like the other cases in Table 1. 


\subsection{Design of Propagation Mode in the Ge Waveguides}

The coupling points of the Ge waveguide with the Si rib waveguide are shown in Figures 2 and 3. The wedge-shaped Ge waveguide coupling with the Si rib waveguide should virtually generate a graded index structure to avoid unwanted scattering loss in terms of a sudden index change that the propagation mode experiences. It has so far been assumed that the propagation mode in the Ge waveguide should be in steady state. However, FDTD simulations of the mode propagation in the Ge and Si waveguide coupling indicate that the propagation mode is not in steady state but is transient in the 5- $\mu \mathrm{m}$-long Ge waveguide, as shown in Figure 3. In other words, the mode bounces ups to the Ge middle and down to the Ge bottom or the Si rib underneath. Accordingly, the propagation mode should experience an in-depth profile of the stress field accumulated in the Ge waveguide, as shown in Figure 2c.

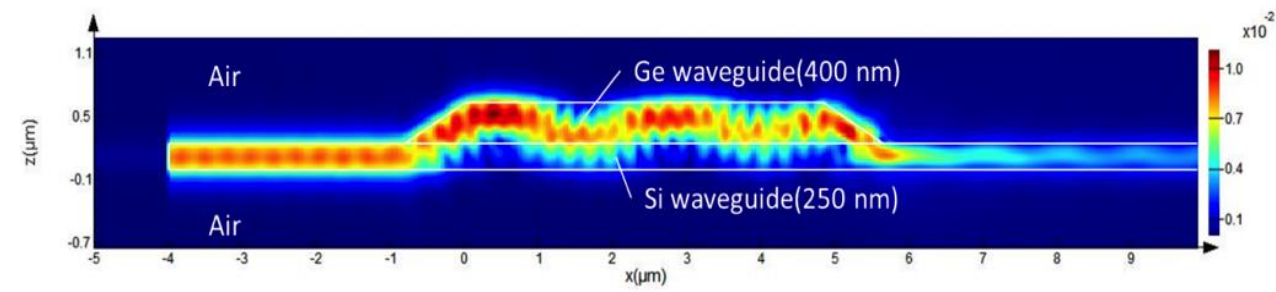

Figure 3. FDTD simulation of the propagation mode of $1550 \mathrm{~nm}$ in the wedge-shaped Ge waveguide on the Si rib waveguide.

Considering that the propagation mode is transient, the transmittance $\mathrm{T}$ of the mode through the Ge waveguide should be expressed by:

$$
T=I_{0}\left(1-e^{-\iint \alpha(s(x, z)) \times L(x, z) d x d z}\right)
$$

Here, $I_{0}$ denotes the incoming power of the propagation mode into the Ge waveguide, $\alpha$ the absorption coefficient given in the Appendix, $s(x, z)$ the stress in the Ge waveguide as a function of the length $(x)$ and the height $(z)$ of the waveguide, as shown in Figure $2 \mathrm{c}$, and $L(x, z)$ the trajectory of the propagating mode in the Ge waveguide, as shown in Figure 3. The rigorous solution of the transmittance has not been obtained from Equation (1), but the red-shift amount at the Ge bottom would be smaller than the prediction in Table 1 . We will discuss on it later in Section 5.

\section{Fabrication of Ge Waveguide on the Si Beam}

Fabrication of the $20 \times 30 \mu \mathrm{m}$ beam ends up with a large removal of the surrounding BOX layer under the Si slab. A hydrofluoric acid (HF) solution is used to etch the BOX layer under the Si beam, which also removes the same amount of the BOX layer under the Si slab, referred to as the "undercut". Bending the beam with a large undercut defocuses stress through the whole structure on the undercut and stress on the Ge waveguide is reduced. To make the undercut small, the beam is designed with many small through-holes $0.5 \mu \mathrm{m}$ in diameter and arrayed every $2.5 \mu \mathrm{m}$ in a square lattice. FDTD simulations suggest that the propagation mode in the $\mathrm{Si}$ rib waveguide would couple to the top of the base $\mathrm{Si}$ wafer, when the beam touches down to the base $\mathrm{Si}$, coupling in radiation mode. To isolate the propagation mode even at touching down, the BOX layer is partially left at the top of the base $\mathrm{Si}$, and thus $\mathrm{SiO}_{2}$ prevents the propagation mode from coupling to the base $\mathrm{Si}$.

To achieve the wedge-shaped Ge waveguide edges, selective epitaxial growth (SEG) of Ge on Si has been used. The $<311>$ faceted mesas on the sidewalls appear at the edge of the SEG Ge, which aligned to the $\{110\}$ planes. The angle between the facet and (100) plane is $25.2^{\circ}$, which should be small enough to form graded index structures at the Ge waveguide, as shown in Figure 3. Thus, the stressing direction should also be in parallel to the $<110>$ directions, as shown in Figure 1. 
The Ge waveguides at the Si beam necks were devised on the top Si layer, using electron beam lithography and reactive ion etching. The 400-nm-thick Ge layer was first grown by an ultra-high-vacuum chemical vapor deposition method. $\mathrm{A} \mathrm{SiO}_{2}$ layer was used as the SEG Ge mask to form (311) faceted wedge-shaped Ge waveguide edges. Reactive ion etching was performed by a $\mathrm{Cl}_{2}$ and $\mathrm{O}_{2}$ gas mixture plasma to fabricate a $400-\mathrm{nm}$-thick Ge waveguide and 150-nm-thick Si rib waveguide.

\section{Results and Analyses}

Figure 4 shows a typical SEM micrograph of the fabricated Si beam. The Si beam with two necks, the $\mathrm{Si}$ rib waveguide with a $10 \mu \mathrm{m}$ bending radius on the beam, and two Ge waveguides on the Si rib waveguide on the necks are clearly shown, as designed. The white "dot" array on the beam is the through-holes to remove the BOX layer for fabrication of the free-standing beam and to maintain a thin $\mathrm{SiO}_{2}$ layer under the beam for optical isolation.

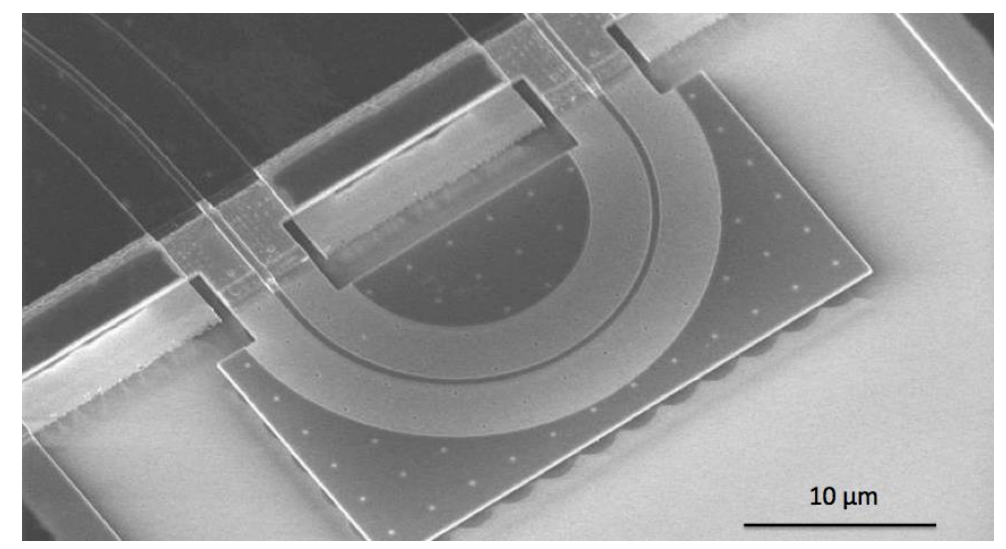

Figure 4. SEM micrograph of the Si beam with two necks. The curved Si rib waveguide and the Ge waveguide on each neck are clearly observed. The many small (white) dots observed on the Si beam are the through-holes to provide a HF solution under the beam.

Figure 5 shows transmission spectra of the Ge waveguide under no stress (green) applied to it other than that applied to the "Touch" (red) and "Deep" (blue) points, as defined in Figure 1a. The curve fittings are performed based on the calculation of the absorption coefficient, due to the Ge indirect bandgap that is calculated using one phonon model [19]. The details are described in the Appendix. The fittings well reproduce the experiment results, where the temperature-dependent constant at $291 \mathrm{~K}$ is used at $1900 \mathrm{~cm}^{-1}$ for the best fits shown in the Appendix. It is experimentally shown from the results that the red-shift by stressing is $\sim 7 \mathrm{~nm}$ at "Touch", and $\sim 52 \mathrm{~nm}$ at "Deep", assuming $2 \times 5$ - $\mu \mathrm{m}$-long Ge waveguides on the necks. Here, the beam was bent by applying an external force to touch down to the base Si to get these red-shifts. The absorption curve at "No stress" was used as the reference.

These observations indicate that the absorption edge at the propagation mode is to experience is $\Gamma-\mathrm{HH}$ as shown in Figure 1. It may be fair to exclude $\Gamma$ - $\mathrm{LH}$ since the red-shifts are too large $(35 \mathrm{~nm}$ and $200 \mathrm{~nm}$ at the "Touch" and "Deep" points). The red-shifts by $\Gamma-H H$ as shown in Table 1 are $15 \mathrm{~nm}$ and $90 \mathrm{~nm}$ at stressing the "Touch" and "Deep" points, which are twice larger compared with the observations. The discrepancy is due to the fact that the propagation mode is transient and the stress profiles in the depth and along the waveguide are inhomogeneous. It is rather hard to predict how the transmission will behave. We will come back to this point in the discussion. Here, it is concluded that applying dynamic stress to the Ge waveguide on the Si beam could precisely tune its fundamental absorption edge at any wavelength in the range of $40 \mathrm{~nm}$. In other words, Ge EAMs, if fabricated on 
the beam, will cover the entire C-band range $(35 \mathrm{~nm})$ in the optical communication wavelength by dynamic stressing at the "Deep" point.

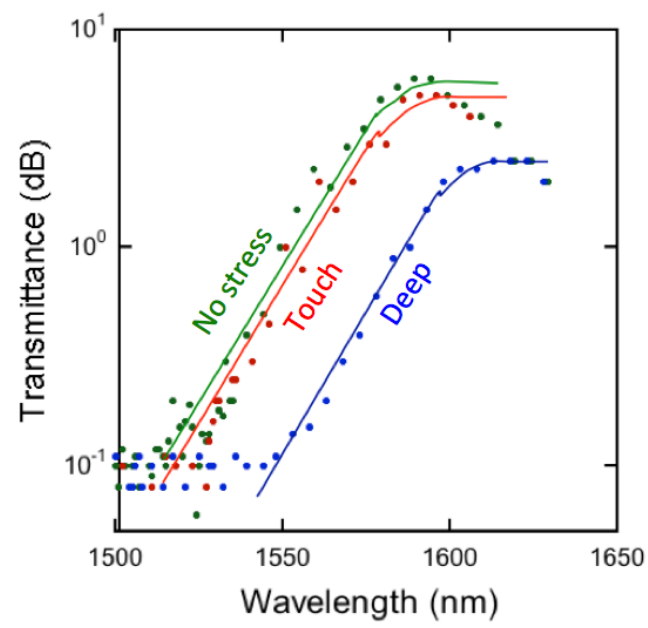

Figure 5. Transmission spectra of the Ge waveguide on the Si beam neck. The green denotes "No stress", the red "Touch" and the blue "Deep", as in Figure 1a. A small red-shift is detected when stress is applied to the "Touch" point, while larger red-shift is detected when stress is applied to the "Deep" point. The solid lines are the fitting results using the one photon model calculation described in the Appendix.

\section{Discussion}

To understand the discrepancy between experimental transmittances in Figure 5 and theoretical predictions made by Equation (1), first we should assume that the trajectory of the propagating mode in the Ge waveguide does not change upon the strength of stressing. In other words, it is always the same as the one shown in Figure 3. We can define $L(x, z)$ in Equation (1) as a sinusoidal curve; the period along the $x$ direction is $2 \mu \mathrm{m}$ and the intensity is half of the Ge waveguide height $(\mathrm{H})$ with $\mathrm{z}=0$ at the $\mathrm{Ge}$ and $\mathrm{Si}$ interface. We further assume that the stress along the Ge waveguide direction is uniform although actual distribution exists, as shown in Figure 2c. This leads us to express Equation (1) as:

$$
T=I_{0}\left(1-\int d x e^{-\alpha\left(s_{\max }(\sin x)\right) \times \mathrm{H} \sin x}\right)
$$

Here, $s_{\max }$ is the maximum stress the mode field experiences depending on the stress point. The point should be the Ge middle. A simple calculation using Equation (2) showed us that the red-shift of the fundamental absorption edge becomes $7.5 \mathrm{~nm}$ at "Touch" and $45 \mathrm{~nm}$ at "Deep". These agree with the observed red-shifts in Figure 5, i.e., $7 \mathrm{~nm}$ at "Touch" and $52 \mathrm{~nm}$ at "Deep", despite the fact that the non-uniformity of stress along the Ge waveguide direction as shown in Figure $2 \mathrm{c}$ is ignored in this calculation. This leads us to conclude that the discrepancy between the predicted red-shifts in Table 1 and the observed ones in Figure 5 should be induced by the transient propagation mode in the Ge waveguides.

The transmittance results in Figure 5 show clear reductions at the wavelength range beyond $1600 \mathrm{~nm}$, despite the fact that the theoretical curves do not show such decreases. The difference can also be explained by the transient propagation mode in Figure 3. The propagation mode smoothly couples down to the Si rib waveguide as shown in Figure 5. It should be noted that the wavelength is $1550 \mathrm{~nm}$ in the FDTD simulations. The propagation mode should hit the (311) Ge waveguide wedge when the wavelength becomes different from $1550 \mathrm{~nm}$. This is the reason why the transmittance is reduced beyond $1600 \mathrm{~nm}$. Such a reduction should have happened to the wavelength range around 
$1500 \mathrm{~nm}$. However, the intensities of the transmittance are below the noise level of these measurements and thus it is not detectable.

Finally, it should be proposed that the stress tuning of the Ge waveguide could be done using an electrostatic force applying a voltage across the beam and the base Si wafer [20]. By fabricating an electrode at "Deep" on the Si beam and applying a voltage across the electrode and the base Si to touch the "Deep" point down to the base Si, the $52 \mathrm{~nm}$ red-shift should be reproduced. In this configuration, the $\mathrm{SiO}_{2}$ layer intentionally left on the base $\mathrm{Si}$ should work for isolating the Si beam not only optically but also electrically from the Si wafer.

\section{Conclusions}

The present paper has proposed a way to dynamically tune the fundamental absorption edge of Ge waveguides by applying tensile stress to them and it described a prototype Ge waveguide on a Si beam structure. The three-dimensional structures of microelectro-mechanical systems (MEMS)-based Ge waveguide on a Si beam are optimized in terms of stress, mechanical stability, and propagation mode and then fabricated. It has been demonstrated that the absorption edge of two Ge waveguides on the Si beam can be dynamically tuned to show the red-shift in the wavelength range of $\sim 52 \mathrm{~nm}$ by applying an external force of 1 GPa to the "Deep" point of the Si beam. The experimentally observed red-shifts are well explained, considering the transient mode of propagation in the Ge waveguides. The presented dynamic tuning method should enable the reconfiguration of the operation wavelength range of Ge electro-absorption modulators in the entire C-band. We propose that an electro-static force can be applied to achieve on-chip stress tuning devices in practical applications.

Acknowledgments: This research project was partly supported by the Ministry of Education, Culture, Sports, Science and Technology (MEXT), Japan (Nanotechnology Platform, Project No. 12024046).

Author Contributions: M.H. and L.M.N. designed and performed the experiments; H.F. performed electro-static tuning experiment; Y.I. and K.W. co-supervised the studies.

Conflicts of Interest: The authors declare no conflict of interest.

\section{Appendix}

In the one phonon model [19], the indirect absorption has the quadratic dependence in the energy difference between the photon energy on one hand and the band gap energy plus or minus phonon energy.

$$
\alpha=A\left[\frac{1}{1-e^{-\Theta / T}}\left(\frac{\hbar \omega-E_{G . I n d}-k \Theta}{h v}\right)^{2}+\frac{1}{e^{\Theta / T}-1}\left(\frac{\hbar \omega-E_{G . I n d}+k \Theta}{h v}\right)^{2}\right]
$$

Here, $A$ denotes a temperature-dependent constant $\left(\mathrm{cm}^{-1}\right), k \Theta$ the phonon energy, $\hbar \omega$ the energy of the photon, $E_{G \text {.Ind }}$ the indirect band gap $(0.664 \mathrm{eV})$ and $T$ the temperature in Kelvins. For simplicity, we use the longitudinal acoustic phonon of Ge with the energy of $27.7 \mathrm{meV}$. The built-in stress in the Ge epilayer on Si splits the valence band into the $L H$ and the $H H$ band, as in Figure 1 . The contribution of each indirect band to the total absorption, $\alpha$ (total), depends on the effective masses and can be formulated as follows:

$$
\alpha(\text { total })=k_{1} \alpha(L H)+k_{2} \alpha(H H)
$$

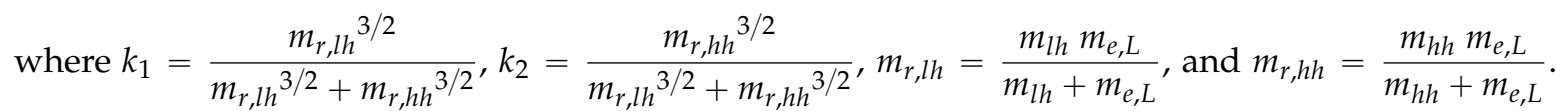
The electron mass at $L$ valley $m_{e, L}$ is $0.22 \mathrm{~m}_{\mathrm{O}}$. Using Equations (A1) and (A2), we obtained Figure 4 to fit the experimental data. 


\section{References}

1. Green, W.M.; Rooks, M.J.; Sekaric, L.; Vlasov, Y.A. Ultra-compact, low RF power, 10 Gb/s silicon Mach-Zehnder modulator. Opt. Express 2007, 15, 17106-17113. [CrossRef] [PubMed]

2. Reed, G.T.; Mashanovich, G.; Gardes, F.Y.; Thomson, D.J. Silicon optical modulators. Nat. Photonics 2010, 4, 518-526. [CrossRef]

3. Xu, Q.; Schmidt, B.; Pradhan, S.; Lipson, M. Micrometre-scale silicon electro-optic modulator. Nature 2005, 435, 325-327. [CrossRef] [PubMed]

4. Watts, M.R.; Zortman, W.A.; Trotter, D.C.; Young, R.W.; Lentine, A.L. Vertical Junction silicon microdisk modulators and switches. Opt. Express 2011, 22, 21989-22003. [CrossRef] [PubMed]

5. Jongthammanurak, S.; Liu, J.F.; Wada, K.; Cannon, D.D.; Danielson, D.T.; Pan, D.; Kimerling, L.C.; Michel, J. Large Electro-optic Effect in Tensile Strained Ge-on-Si Films. Appl. Phys. Lett. 2006, 89. [CrossRef]

6. Liu, J.F.; Pan, D.; Jongthammanurak, S.; Wada, K.; Kimerling, L.C.; Michel, J. Design of monolithically integrated GeSi electro-absorption modulators and photodetectors on a SOI platform. Opt. Express 2007, 15, 623-628. [CrossRef] [PubMed]

7. Liu, J.F.; Beals, M.; Pomerene, A.; Bernardis, S.; Sun, R.; Cheng, J.; Kimerling, L.C.; Michel, J. Waveguide integrated, ultra-low energy GeSi electro-absorption modulators. Nat. Photonics 2008, 2, 433-437. [CrossRef]

8. Lim, A.E.; Liow, T.; Qing, F.; Duan, N.; Ding, L.; Yu, M.; Lo, G.; Kwong, D.-L. Novel evanescent coupled germanium electro absorption modulator featuring monolithic integration with germanium PIN photodetector. Opt. Express 2010, 19, 5040-5046. [CrossRef] [PubMed]

9. Feng, N.N.; Feng, D.; Liao, S.; Wang, X.; Dong, P.; Liang, H.; Kung, C.C.; Qian, W.; Fong, J.; Shafiiha, R.; et al. $30 \mathrm{GHz}$ Ge electro-absorption modulator integrated with $3 \mu \mathrm{m}$ silicon-on-insulator waveguide. Opt. Express 2011, 19, 7062-7067. [CrossRef] [PubMed]

10. Feng, D.; Liao, S.; Liang, H.; Fong, J.; Bijlani, B.; Shafiiha, R.; Luff, B.J.; Luo, Y.; Cunningham, J.; Krishnamoorthy, A.V.; et al. High speed GeSi EA modulator at 1550 nm. Opt. Express 2012, 20, 22224-22232. [CrossRef] [PubMed]

11. Nguyen, L.M.; Kuroyanagi, R.; Tsuchizawa, T.; Ishikawa, Y.; Yamada, K.; Wada, K. Stress tuning of the fundamental absorption edge of pure germanium waveguides. Opt. Express 2015, 23, 18487-18492. [CrossRef] [PubMed]

12. Kuo, Y.-H.; Lee, Y.K.; Ge, Y.; Ren, S.; Roth, J.E.; Kamins, T.I.; Miller, D.A.B.; Harris, J.S. Quantum-confined stark effect in Ge-SiGe quantum wells on Si for optical modulators. IEEE J. Sel. Top. Quantum Electron. 2006, 12, 1503-1513. [CrossRef]

13. Chaisakul, P.; Marris-Morini, D.; Roulfed, N.-S.; Frigerio, J.; Charastina, D.; Coudevylle, J.-P.; Roux, X.L.; Edmong, S.; Isella, G.; Vivien, L. Recent Progress in GeSi electro-absorption modulators. Sci. Technol. Adv. Mater. 2014, 15, 14601-14608. [CrossRef]

14. Ishikawa, Y.; Wada, K.; Cannon, D.D.; Liu, J.; Luan, H.-C.; Kimerling, L.C. Strain-induced band gap shrinkage in Ge grown on Si substrate. Appl. Phys. Lett. 2003, 82. [CrossRef]

15. Yoshimoto, K.; Suzuki, R.; Ishikawa, Y.; Wada, K. Bandgap control using strained beam structures for Si photonic devices. Opt. Express 2010, 18, 26492-26498. [CrossRef] [PubMed]

16. Cai, J.; Ishikawa, Y.; Wada, K. Strain induced bandgap and refractive index variation of silicon. Opt. Express 2013, 21, 7162-7170. [CrossRef] [PubMed]

17. Horie, Y.; Decostered, L.; Suzuki, R.; Ishikawa, Y.; Wada, K. Emission wavelength tuning by mechanical stressing of GaAs/Ge/Si microbeams. Opt. Express 2011, 19, 15732-15738. [CrossRef] [PubMed]

18. Wada, K.; Yoshimoto, K.; Horie, Y.; Cai, J.; Lim, P.H.; Fukuda, H.; Suzuki, R.; Ishikawa, Y. Srtrained Ge for Si-Based Integrated Photonics. In Photonics and Electronics with Ge; Wada, K., Kimerling, L.C., Eds.; Wiley-VHC: Weinheim, Germany, 2015; p. 219. 
19. Macfarlane, G.G.; Roberts, V. Infrared Absorption of Germanium near the Lattice Edge. Phys. Rev. 1955, 97. [CrossRef]

20. Fukuda, H. A Study of Silicon Microphotonics for Telecommunication Applications. Ph.D. Thesis, the University of Tokyo, Tokyo, Japan, 2014.

(c) 2016 by the authors; licensee MDPI, Basel, Switzerland. This article is an open access article distributed under the terms and conditions of the Creative Commons by Attribution (CC-BY) license (http://creativecommons.org/licenses/by/4.0/). 